\title{
Barcelona/s: tres relatos y un poema con mujeres en el balcón
}

\author{
JUAN M. RIBERA \\ (UCM) \\ jumriber@filol.ucm.es
}

\begin{abstract}
Resumen: Aproximación a las escripturaciones literarias de la ciudad de Barcelona según los códigos culturales masculino y femenino. Ejemplificación de la incorporación de la mujer a las letras catalanas contemporáneas, mediante la atención a tres diversos niveles de presencia y participación en la realidad humana y urbana de Barcelona así como de su captación literaria por parte de escritoras señeras de los siglos XIX y XX.
\end{abstract}

Palabras clave: Literatura catalana contemporánea. Estudio de género. Barcelona, autores y autoras. Jacint Verdaguer, Joan Maragall y Joan Oliver Pere Quart. Dolors Monserdà, M. Teresa Vernet, Montserrat Roig y MariaMercè Marçal.

\begin{abstract}
This paper is an approach to the conversion of the city of Barcelona into literary material according to male and female cultural codes and it exemplifies women's insertion in contemporary Catalan literature by addressing three different levels of presence and participation in Barcelona's human and urban reality and its literary perception by *leading* women writers of the 19th and 20th centuries.
\end{abstract}

Key words: Contemporary Catalan literature. Gender study. Barcelona, male and female authors. Jacint Verdaguer, Joan Maragall and Joan Oliver - Pere Quart. Dolors Monserdà, M. Teresa Vernet, Montserrat Roig and MariaMercè Marçal.

Más tres odes, habrá que añadir a la segunda parte del título y por lo que de inmediato se aclarará; y aún cabe la advertencia de que pasamos a tratar la aparición - y posesión- de diversas barcelonas de mano de las mujeres como actantes y con letra de mujeres como autoras, acatando su acoso, toma y gradual codificación de la ciudad que las vio nacer como evidencia intercultural, en este caso y desde los presupuestos de los estudios de género y de la ginocrítica por su diversa percepción del entorno vital desde un sistema distinto del social y cul- 
turalmente masculino. Es para marcar esta diferencia para lo que acudimos, a modo de incipit, a las mencionadas odes dedicadas a Barcelona y sucesivamente compuestas por Jacint Verdaguer, en 1883 (Pàtria, 1888), Joan Maragall, en 1909 (Seqüències, 1911) y Joan Oliver - 'Pere Quart', en 1936 (ed. por Comissariat de Propaganda de la Generalitat de Catalunya).Tras ese preámbulo, acabaremos de informar, se pasará con más detenimiento por cuatro escripturaciones de Barcelona, debidas a cuatro autoras crecidas en diferentes contextos socioculturales y pertenecientes a diversas promociones literarias: Dolors Monserdà, Maria Teresa Vernet, Montserrat Roig y Maria-Mercè Marçal. Los ordenados pasos entre sus documentos evidencian un recorrido favorable a aquella toma y codificación de Barcelona por parte de la mujer. Proponemos un itinerario entre una cartografía más amplia, la que queda documentada en el volumen Barcel/dones (1989) que aquí proponemos como enriquecedor telón de fondo para las líneas que siguen.

1. Jacint Verdaguer (1845-1902), Joan Maragall (1860-1911), Joan Oliver 'Pere Quart' (1899-1986): Tres odes masculinas a un cuerpo femenino.

Destáquese brevemente cómo, desde su inherente calidad poética, las tres odes forjan, con toda seguridad entre otros niveles semánticos significativos, una corporeidad de Barcelona sustentada en su feminidad ante actantes masculinos. Así, desde el fervor romántico renaixencista, Jacint Verdaguer (1974: 407-412) visiona épicamente una ciudad que es «filla» del monte sagrado que la tutela, el Montjuïc (est. I), que pare linajes de sus «entranyes» (est. II) y que guía maternalmente a sus hijos (est. III); reverenciada por una mar, «la mar», si nació «amazona» ha acabado por ser «matrona» (est. IV-VI), velada por otros montes hijos del Pirineo, círculo-cuna de su crecimiento, el que anima la urbe o «mare» de las barriadas que genera y de las cercanías que absorbe (est. VII-XV), hasta el linde de los pueblos costeros que semejan «nimfes catalanes» (est. XVI), adoradoras de quien se nos representa como una Venus nacida de las aguas $\mathrm{y}$ «filla» $\mathrm{y}$ «pubilla» de la patria catalana (est. XX-XXIII); mater generatrix del progreso (est. XXII) y de tantos héroes y sabios varones - tan sólo, ai!, encomendada a una presencia femenina, la patrona Santa Eulària- (est. XXIV-XXIX), es «jardí»o tierra sembrada de templos y monumentos (est. XXIX-XL), es «reina» del mar ante una historia usurpada y «aurora» de nuevos tiempos; y, claro está, «ciutat dels Comtes», perpetuación de una figura, Clemència Isaura, descendiente de los fundacionales condes de Tolosa (est. XLI-XLVI). Sólo aquella advocación religiosa y esta última mención histórica que nos acerca a un apéndice femenino del linaje masculinamente fundacional nominalizan a dos mujeres en un espacio que rezuma naturaleza femenina por los cuatro costados pero para ser escenario de presencias orográficas e históricas masculinas. Para Jacint Verdaguer, Barcelona-hija-doncella cualificada-madre es útero de una heroica historia descifrada en claves masculinas. Cabe argüir que la propia voz Barcelona impone una personalización femenina; así mismo que en el genérico «poble» cristiano (est. XLIV) que, según se canta, ocupa aquel magma urbano se engloba población de ambos géneros. Con todo, cuesta entender que la ciudad en esencia poetizada por Verdaguer como regazo y vientre únicamente acoja dos presencias femeninas que no son sino representantes de dos códigos — tradición religiosa e historia política— que, jugando a favor de los hombres, han sido controlados por los varones. 
Ante tal iconografía en un texto que no deja de alentar una tradición lírica en torno a Barcelona como motivo poético pero habiendo pasado la urbe y la sociedad catalanas por la experiencia traumática de la Setmana tràgica (1909), Joan Maragall atiende una ciudad en crecimiento o tal vez huída hacia el horizonte pirenaico (est. I-II), el mismo manto cobijador del que derivaban las estribaciones montañosas cantadas por Verdaguer. Pero lo hace para detenerla y advertirla mediante un conminador diálogo (est. III-VI) y, desde los versos siguientes y hasta el final de la composición (est. VII-XV), para amonestarla y, no obstante, a la postre rendirse enamorado y reconocerse esclavo suyo. En ese largo período, Maragall perfila una Barcelona de buena «planta» a la vez que «grollera», «riallera» no menos que «vanitosa», tan «traïdora» como propicia a desdecirse; en suma, toda una serie de rasgos que transmite el tópico de la volubilidad femenina proyectado sobre la mujer-ciudad reconvenida. Una protagonista con sus calles, barriadas y ramblas, cierto es, en ese momento abandonada al «incendi», al «vilipendi», pero también a la «sang» y a la «discòrdia»; salvada, no obstante, por un «cel» pacificador y por un «místic temple»-la Sagrada Familia—, por unos signos operativamente masculinos, neutralizadores de esa «ciutat mala» y «...vana i coquina i traïdora i grollera» que el poeta, con todo, adora: «Tal com ets, tal te vull, ciutat mala», «Barcelona nostra!» pero, no menos, «la gran encisera».

Poema que cabría leer junto a los artículos maragallianos suscitados por las obras del templo de Antoni Gaudí y por los dramáticos hechos de la revuelta mencionada -entre El Temple que naix (1900) y La ciutat del perdó (1909)—, también contando con la comprensión de la gran ciudad como la mayor de las prostitutas de acuerdo con la imagen proyectada desde el París finisecular e importada por los modernistas, una y otra hipótesis ponen ante Barcelona a un yo masculino abducido por los encantos y los peligros, por la seducción vaginal de un cuerpo femenino que ya no es únicamente madre; es una hechicera amante de cuyos poderes sólo un dios masculino podrá salvar al varón. Posibilidad ante la cual, creemos, este último nunca llegará a renunciar del todo a su querida, reconociéndose por siempre seducido, incluso en el caso del cristiano poeta que fuera Joan Maragall.

Díptico barcelonés, de una a otra oda, ante el que Joan Oliver se posiciona, en tiempos de guerra, junto con una Barcelona herida bajo los bombardeos (d. est. I). Es una Barcelona que, si no deja de ser una «bruixa frenètica», se muestra ahora desvergonzada y con estridente maquillaje, al fin abandonada por sus frívolos amantes de antaño, de los finiseculares tiempos de gloria (est. VIIVIII). Es, en suma, aquella amante hechicera convertida en una ajada prostituta que avanza ahora entremezclada entre el desorden y el gentío popular de un nuevo paisaje histórico, por entre signos desvencijados de un tránsito de siglos ya caducado (est. IX-XI). Una vieja criatura, cierto es, a la que se concede un horizonte, imprecando a Barcelona de estrofa en estrofa (est. XII-XVIII): acompañada por una mar femenina — aquella «la mar» fiel que viene de los versos maragallianos-, se le vislumbra una «renaixença», un futuro capitalino y nacional, en que podrá llegar a ser «mestressa sobirana», siempre que no se abandone a encomiásticos cantos - y aquí Oliver se aleja de la perspectiva de Verdaguer y de Maragall, sí habiendo coincidido con el vate modernista en cuanto a 
la visión destruida de su urbe en dos distintas circunstancias-; siempre, abunda 'Pere Quart', que aquella ciudad-criatura tome el pulso a su propio «cor», que sepa estar en la línea de partida del futuro que comienza, atenta y dispuesta a tomar las riendas de la historia que se inicia. En ese punto, se aprecia el reconocimiento de un ente femenino que, desde su propia naturaleza sexual y de cara a ese porvenir, pudiera engendrar, seres de uno y otro sexo, protagonistas del amanecer previsto, ciudadanía total comprometida con los ideales que defiende el poeta cívico. Oliver-'Quart', frente a los dos textos anteriores, alienta ese paso que finalmente obstruye con el verso final. Si bien pudiera hacerse la lectura de que el genérico hijo remite a aquel bienaventurado porvenir, allí y según nuestra opción, Barcelona vuelve a ser vientre generador de varones y madre condicionada por las atenciones debidas a los actantes prioritarios de la historia: «Pensa en el fill que dus a les entranyes».

Las tres odes, de acuerdo con la lectura propuesta, trazan el itinerario seguido por un cuerpo femenino siempre sujeto al protagonismo excluyentemente masculino. Advertiremos que si pasamos a la narrativa de un tiempo histórico y literario coincidente con el de esas tres composiciones, los narradores parecen más propicios a dibujar una cartografía barcelonesa por donde transitan protagonistas femeninas. Entre Pilar Prim (1906) de Narcís Oller, Fanny (1929) de Carles Soldevila o Vida privada (1932) de Josep M. de Sagarra, progresa un interesante arco a su favor. Por entre el mismo discurren diversas mujeres, alguna de las cuales incluso se expresa mediante su mente y voz, merced al uso del monólogo interior, por primera vez empleado en las letras hispanas por Carles Soldevila. Pero ni siquiera este último y notable ejercicio de travestismo creativo ni justifica la ausencia ni sustituye la presencia de hijas literarias de Barcelona cantadas y contadas por sí mismas. Es a favor de constatar esa incorporación histórico-literaria y también la mencionada toma de Barcelona por sus escritoras, por lo que proponemos atender a los eslabones de los apartados siguientes.

2. Dolors Monserdà (1845-1919), La fabricanta. Novel-la de costumns barcelonines (1860-1875) (1904): Hasta el balcón.

Escritora crecida en la Renaixença catalana de cronología fundamentalmente decimonónica, en su caso y desde 1870 documenta la incorporación de la mujer a las letras catalanas contemporáneas. Dolors Monserdà, a su vez y no obstante, evidencia junto con casos como, por ejemplo, el de Maria del Pilar Maspons - 'Maria de Bell-lloc', la limitación por parte de las escritoras coetáneas a la hora de construir un sistema referencial y literario distinto del estereotipado por las firmas masculinas y en el que incluso la figura de la mujer tiene una parcela de actuación adjudicada y un lenguaje concedido que esas otras firmas femeninas no modifican. Por su protagonismo cultural y su longevidad literaria, Dolors Monserdà podrá ostentar la función de $m(p)$ atriarca de las escritoras en lengua catalana; imposible resultaría adjudicarle la de figura matriz-motriz que, mediante afortunada denominación debida a la Dra. H. Hurtado, muchos reservamos a la modernista Caterina Albert i Paradís - 'Víctor Català', a la hora de reconocer la evidencia y el arranque de una escritura de mujer en catalán que enmendara sustitutoriamente la referida topificación masculina. 
Una escritora, Albert-Català, que, desde el prioritario marco rural de su narrativa, también se adentró en la realidad urbana barcelonesa: su novela Un film (3000 metres) (1926) pasa a instalarse, desde la provincia, en la capital; entendido este destino como moderna «terra de promissió» (Català, 1985: 62), la autora potencia ante él el deslumbramiento por su monumentalidad, aprecia el margen despersonalizador de sus masas, atiende al catálogo de los lugares por donde pasan los hábitos urbanos y lo conjura y hasta descubre su poética mediante una consecuente retórica fragmentarista y de planos cruzados; todo ello vivido, aún contando con el protagonista masculino Nonat, por un personaje colectivo engullido por aquel vientre ocultador del secreto que cada uno de sus componentes necesitaría desvelar para, así, alcanzar su reconocimiento individual; colectividad en la que no faltan secundarios protagonismos femeninos arrastrados por la corriente urbana, sólo el que Víctor Català no haya optado en este título por una primordial protagonista femenina nos ha llevado a desestimar su elección para una de las cuatro calas de estas páginas.

Hecha esta salvedad y no obstante, partimos del mencionado título de Dolors Monserdà con protagonista femenina, Antonieta, que, con todo, no sólo prueba la existencia de la mujer en la urbe barcelonesa sino también su participación en la puesta en funcionamiento de aquella máquina socio-económica, eso sí, desde la trastienda, desde la actuación filtrada y ocultada por y tras el actante masculino. Aún así, resultaría interesante contrastar esa Antonieta con la Toneta protagonista de La papallona (1882) de Narcís Oller y, si bien leeríamos cómo ambas recorren las calles de Barcelona, habríamos de acatar los diversos niveles del discurso en que sus autores ubican a una y a otra, también el diferente alcance de sus actuaciones, de modo respectivo, sobre la vida colectiva o sobre la tragedia de sus sentimientos individuales.

Advertido ese horizonte a favor de Antonieta, sepamos no menos objetivamente dónde la ubica Monserdà y cuál es el radio de acción que le concede en la topografía de su ciudad: la protagonista es vecina crecida en el domicilio familiar del estrecho carrer Sant Pere Mitjà — junto a Pl. Sant Pere, zona que en las fechas de la escritura y de la acción de la novela aún no estaba desmembrada del conjunto del barrio gótico por la posterior apertura de la Via Laietana (19071926), obra que supuso la piqueta para construcciones medievales y el levantamiento de edificios modernistas, así la emblemática sede central de la Caixa de Pensions (1914-1917) trazada por Enric F. Sagnier, emblema de los nuevos tiempos—; es educada en el colegio religioso de las «monges de l'ensenyança quan aquestes tenien encara el seu convent en el centre de la ciutat antiga» (Monserdà 1992: 89) y, bajo la ordenación impuesta por su hermano, empresario además de la firma textil heredada, lo uno y lo otro tras la muerte de sus padres, se mueve entre las obligaciones domésticas y una escasa red de relaciones, limitadamente abierta al balcón donde cuida de sus plantas y de su canario; el mismo y reservado espacio desde donde Antonieta ve pasar a su enamorado, en cuya historia de amor se ve confirmada desde el confesionario y el altar de la cercana iglesia de Sant Pere, también desde el domicilio del pariente sacerdote. No obstante, hasta de ese tradicional ámbito para una mujer de clase media en una ciudad española de finales del ochocientos, resulta expulsada la protagonista por el hermano cuando sabe de su proyecto matrimonial con Pere-Joan, un sim- 
ple «veler» o tejedor de velos. Esa actitud de revuelta por parte de la mujer que piensa en reordenar su vida en función de sus sentimientos, es pagada por Antonieta con la salida sin dote del domicilio familiar y con la prohibición de casarse en aquella su parroquia en calidad de borde repudiada por el hermano. Pero, eso sí, con los enseres maternos con los que ordenar su nuevo hogar y con los artesanales utensilios paternos con los que fundar una nueva fábrica; unos y otros serán el esencial «parament»y los fundamentales «fonaments» (Monserdà 1992: 133,142) de sus dos nuevas y la vez continuistas ubicaciones. La palabra de la protagonista es la que marca un antes y un después en su trayectoria que ya es la de su matrimonio; y esto justo en el episodio de la noche de bodas (Monserdà1992: cap. VIII, 139-143), cuando, ante su consigna de forjar un nuevo futuro en pareja, el recién casado reconoce en el brillo de los ojos de la mujer «...la força de la intel-ligència, de l'energia i de la fe en lo pervindre, tot lo que aquella ànima valia» (Monserdà 1992: 142).

A partir de aquí, la progresión argumental es imaginable. La previsión de la mujer y el esfuerzo compartido por los esposos alentará el crecimiento de aquel proyecto en su cambio «De velers a fabricants» según el título del cap. XIII y entre el crecimiento económico barcelonés de la década de los años sesenta del siglo XIX. Destáquese que ese proceso es ejemplarmente contrapuesto a la decadencia y ruina del hermano de Antonieta como empresario y efímero inversor en bolsa; y, además, Antonieta resulta contrailuminada mediante la actuación de su prima y cuñada, Serafina, causa última del hundimiento de la fortuna fraterna. Evidente bovary de segunda generación, este personaje antagonista organiza su vida sobre el patrón de novelas románticas y naturalistas —unas y otras ampliamente denostadas por Monserdà (Monserdà 1992: por ej. 184-185, 189) en el fragor del gusto finisecular catalán por la literatura zolesca-, paseándose, ella sí, por bailes y teatros barceloneses y viajando hasta París. Pero ahí está Antonieta, ajena a tales distracciones de factura urbana, para poner cada cosa en su sitio. Tanto la economía y la entente en el hogar del hermano como la moraleja final de que el modelo matrimonial y económico — sentimientos y sacrificio- por ella conducido es el patrón sobre el que cimentar plausibles modelos de conducta $\mathrm{y}$, a la postre, de progreso social. Téngase aquí presente a Dolors Monserdà como autora de los conservadores ensayos El feminisme a Catalunya (1907) o Estudi feminista (1909). Y, en consonancia con todo ello, no se espere que la actuación de la protagonista se ejecute, valiéndonos de conceptos woolfianos, mediante su irrupción como figura de reconocimiento público; no le cabe serlo porque su demarcación no la propicia una mujer-escritora acreedora de una $h a$ bitación propia, tampoco porque ella misma no puede escapar a las categorías subalternas con respecto a los hombres tal y como se reservaba a la mujer en los relatos de autoría masculina. Antonieta pasa de ser hermana de a asumir el papel de esposa de. Su objetiva conducción de los resortes sobre los que crece el mundo y la ciudad que lo representa en su narración, eso resta sellado en el pacto de la vida doméstica, bajo la sumisión debida como esposa, «menestrala» o verdadera alma de su unidad familiar pero en la sombra. Con esto último comulga fervorosamente Antonieta a modo de verdadera consumación matrimonial mediante sus palabras en el cierre del mencionado episodio de la particular noche de bodas y en ello la confirma la actuación del marido al final del libro 
(Monserdà, 1992: 142-143, 249-251). Ahora bien, en la novela catalana del tránsito de centurias, ese es un primer indicio firmado por una escritora a favor de la toma de la ciudad por parte de la mujer, de su pulsación en el momento de refundarse Barcelona como capitalidad moderna. Urbe ante la cual, cierto es, sólo le estaba permitido asomarse hasta el balcón del domicilio regentado por el hombre. Su capacidad de acción es domésticamente neutralizada, institucionalmente ocultada. Pero, como documento, esa fuerza subterránea nos resulta interesante a modo de umbral para la trayectoria que proponemos como recorrido.

3. Maria Teresa Vernet (1907-1974), Les algues roges (1934): Asomada al balcón.

Escritora editorialmente presente desde 1926, forma parte, durante los años veinte y treinta del novecientos y junto a nombres como Aurora Bertrana, Anna Murià o Mercè Rodoreda, de la promoción de autoras que, en las letras catalanas, normaliza el acceso de la mujer a la vida pública a través de la actividad cultural y de su profesionalización literaria. Maria Teresa Vernet, a favor de sus algas rojas - cuya cimbreante imagen y creciente corporeidad bajo las aguas de los estanques es espejo del desarrollo de sus protagonistas a lo largo de su novela, sus Isabel y Marina-, dispone todo un mapa urbano no exento de una significativa estratificación.

La historia cruzada y crecientemente cómplice entre una joven educada en firmes valores y licenciada en Historia por la Universidad de Barcelona y una muchacha marcada por carencias afectivas y materiales, se inicia, in media res, en un club de tenis. Desde ese moderno umbral su relato transcurre, en la primera parte de la novela, por o ante un «niu d'amor», la «platja», un «bar», un «balneari», un «teatre», un «cinema» o las viviendas «als afores» y progresa Diagonal o Passeig de Gràcia adelante; lugares cada uno de ellos donde las mujeres de Vernet, junto a los restantes barceloneses transitan en «tramvia», practican deporte, toman baños de sol, se maquillan y beben y fuman, se divierten y aman, ironizan sobre la moda del freudismo literario o se ríen del sentimentalismo romántico a lo Dama de las Camelias, debaten sobre la virginidad femenina y alientan una red de relaciones emocionales y sexuales. Se trata, en suma, de un medio urbano sustitutorio del tradicional y donde una mujer puede sentir, en la ducha, que «....assaboria la felicitat del seu cos» (Vernet 2006: 4).

La novela, mediante esos referentes, no deja de pasar por una corografía urbana en exceso topificada por la novelística europea de entreguerras. Tópica comprensible ante los márgenes de apuesta por la modernidad que suponía el modelo de cosmópolis crecido desde el cambio de centuria, Vernet hace que Marina, asomada al balcón, abarque ese microcosmos: «Sortí al balcó xiulant i encengué una cigarreta. A baix començava l'animació nocturna; els bars i cinemes i cabarets enlluernaven amb el parpallejar dels anuncis cridaners; els aparadors lluien amb tot l'esclat de les quincalles i les sedes arificials; les dones s'empentaven per les voravies...» (Vernet 2006: 34). Cierto que ese es el mismo animado espectáculo urbano sobre el que aquella protagonista volverá a asomarse horas más tarde para verlo desvanecido. Pero en tal ciudad — realidad efímera, sí, pero ya públicamente suya-crecen, incluso con sus contradicciones, unas mujeres distintas — «Ara qui les entén, les noies? Aquesta, què vol?» 
(Vernet 2007: 37), se interroga la madre de Marina-, entre las cuales, esta última se hunde en una doble historia a caballo de un novio que la mantiene y un amante al que desea. Tal red de relaciones pasada por la atadura al hombre ya no es excluyente de otro tipo de vivencias, practicadas a lo largo y ancho de una urbe desde la que surgen las voces y los contrastes que hacen de Marina un ser insatisfecho. En ese primer tiempo del argumento, la presencia casi latente de Isabel actúa como contra-discurso con el que choca la actuación de Marina.

El paso dado por esta última junto con su amante hacia París, desplaza, ya en la segunda parte de la novela, la acción hacia la ciudad del Sena, destino también para una Isabel que lleva adelante una investigación académica. De modo breve señalaremos que tal desplazamiento geográfico se acompaña además de menciones a Londres, Berlín o Florencia en otro guiño de correspondencia literaria acorde con el indicado con anterioridad, ahora insistiendo en la confianza de estar viviendo una época marcadamente cosmopolita, con preferencia por lo urbano; y aún que la novelista es muy sistemática en cuanto al callejero, los barrios y los monumentos parisinos cuando, en la parte transcurrida en Barcelona, había optado más por denominaciones denotativas que por las puntualmente nominales. En cualquier caso, en ese París se marca el reencuentro entre las amigas, la abandonada por su amante y la jubilosa investigadora; una y otra reconocen sus experiencias - desvelándose una primera relación sexual de Isabel, superada y que la hizo fuerte merced a la propia decisión de descartarla en su proyecto de vida, mientras que Marina había quedado atrapada entre las suyas, malentendidas como vías escapatorias-; en aquel destino refuerzan su complicidad al tiempo que inician unos más estrechos lazos que la Dra. M. Campillo aprecia de naturaleza materno-filial, punto donde se acentúa el didactismo de la autora que en ocasiones la puede haber llevado a condicionar sus estructuras narrativas (Vernet, 2006: XXIX, XXIV); y es allí e instalada en esa perspectiva, donde Isabel transmite a Marina su comprensión femenina de la existencia: «- No has de dir mai mai: sóc sola. Has de dir: sóc lliure!» (Vernet 2006: 122).

Ese es el horizonte geográfico y vital desde el que, ya en la tercera parte de la novela y primero, regresa Marina a Barcelona y, más tarde, Isabel. Una Marina reeducada en su aprendizaje junto a Isabel que ha de afrontar la historia pendiente, dejada atrás en su huída de Barcelona. Se trata de la relación con la madre, el recuerdo lacerante del novio, las subconscientes expectativas de reencuentro con el amante... Apreciamos que, para tal tránsito, no exento de vaivenes por parte de su protagonista, Vernet ha desestimado lugares e itinerarios concretos trazados anteriormente. Marina se mueve ahora entre espacios más abstractos o quizás trascendentes como el domicilio recuperado, el hospital donde está ingresada su madre, la barriada de Horta... Por entre ellos se filtra su último crecimiento como persona madura, reconciliada con su existencia y reencontrada con la amiga regresada de Paris y ante la que va sintiéndose su igual, dispuestas a vivir ambas la madurez de sus biografías, incluso con previsiones en lo sentimental pero no atrapadas por ello. Cabe pensar que el decisivo control por parte de Marina de las riendas de su vida coincide con la definitiva toma de posesión de su ciudad. Así mismo, cáptese que, circularmente, la novela se cierra en el club de tenis, allí donde, ante la pregunta de Isabel y ante una incipiente primavera a la vez que desde uno de los núcleos representativo de la 
nueva urbe codificada en la primera parte de la novela, Marina afirma con voz segura que para nada volvería a su antigua existencia. Con esa coincidencia de discurso y espacio, Maria Teresa Vernet se reafirma doblemente. Lo hace a favor de la ciudad moderna de su generación, tomada y puesta al servicio de un modelo de mujer que puede pasear por entre aquella nueva topografía una existencia a la vez firme y pública. aire.

4. Montserrat Roig (1946-1991), Barcelona a vol d'ocell (1987): Desde el

Firma que, junto a la de escritoras del resto de la geografía lingüísticamente catalana como la valenciana Isabel-Clara Simó o la mallorquina Carme Riera, compone en la vida pública catalana el perfil de la intelectual y profesional de la cultura que, académica o mediáticamente, protagoniza la historia de su tiempo, entre la transición democrática española y el límite del novecientos. Montserrat Roig, a partir de los años setenta y sin haber alcanzado a transitar entre las centurias, conjugó en su prosa la crónica de Barcelona y la historia de su ciudadanía mediante su narrativa, ensayismo, dietarismo y reportajes. Lo dejó hecho desde la toma de conciencia social y política. Compromiso del que no excluyó la conciencia de género y la revisión de la incorporación de la mujer a la cultura catalana; así, parte de su novelística — desde Ramona, adéu (1972) a L'òpera quotidiana (1982), pasando por El temps de les cireres (1977) y L'hora violeta (1980) - se articula sobre sagas familiares cuya trayectorias se siguen atendiendo a los eslabones enlazados por protagonistas barcelonesas. Condición, esta última, que era la suya propia y de las mujeres de su entorno familiar, amistoso y profesional. De acuerdo con esto, en sus páginas hallamos muestras más que suficientes de la presencia de la mujer en el espacio de su ciudad. Cada uno de esos textos evidencia el tiempo y los modos de las particulares tomas de posesión de Barcelona por parte de sus emblemáticas protagonistas, desde finales del ochocientos hasta la década de los setenta vivida por la autora. Y cada una de estas últimas nos regalará con un prisma de un calidoscopio que, en su globalidad, supone la Barcelona asumida y animada por Montserrat Roig.

Preferimos, no obstante esa segura fuente de documentación, optar por otra perspectiva, la aérea, desde donde la palabra de Roig, gestada desde una balconada o mirador si cabe más absoluto que el balcón del texto anterior, pronuncia un particular relato colectivo de su ciudad. Particular, decimos, porque la forma más tradicional de la narración es sustituida por la de la crónica histórica y humana que anima el reportaje sobre Barcelona, meditado por Roig desde el aire al tiempo que Xavier Miserachs disparaba sus panorámicas y por ella compuesto desde el conocimiento del día a día de la longeva urbe mediterránea; y, así mismo, decíamos colectivo al poder abarcar al unísono todos sus horizontes y los de su población. Es de ese modo y desde la baranda más etérea de la avioneta de trabajo como la escritora se plantea objetivar su ciudad; eso sí, tras haberla «...llegida amb els peus i [...] dominada amb el record» y, no menos, llevada por la «...curiositat per a comprendre la meva ciutat des d'una perspectiva nova, alada...» (Roig, 1987: 18, 22). Así da forma literaria al anillo de colinas que circunda Barcelona, a sus trazados góticos o cartesianos y a sus parques, a sus barrios y a sus monumentos, mientras, bajo el aire, con maneras de cuerpo 
sometido a una inmediata autopsia o de amante abandonada a la penetración más devota, «Barcelona té els budells descoberts, i nosaltres estem a punt de fer-hi una dissecció, escorxada als nostres peus, una mica impúdica», «...esventrada i somnolenta» (Roig, 1987: 22, 28). Con tal disposición, Barcelona se ofrece a los ojos y al conocimiento de su vecina como «...una ciutat mediterrània, atapeïda, amb els terrats que ens somriuen i els forats de les illes de l'Eixample, que semblen pollastres farcits d'uralita i de maons» (Roig, 1987: 28).

Aprovecharemos esa primera llamada textual a las «illes» o manzanas y sus patios, trazados por Ildefons Cerdà entre las viviendas del Eixample del que la autora fuera vecina, esos espacios abiertos y originalmente ajardinados, concitadores de la intimidad o de la plácida convivencia en pleno centro urbano y que el tiempo fue llenando de almacenes y de aparcamientos. Acompáñese tal focalización de las fotografías de Xavier Miserachs que, a modo de contrapicado en círculos concéntricos, pasan del plano general a la visión de alguno de esos parajes mayoritariamente anulados, por ejemplo al aún hoy conservado Passatge Permanyer (entre Via Laietana/C. Roger de Llúria-C. Consell de Cent/C. Diputació), «petit oasi de pau i tranquil-litat» (Roig, 1987: 128-129, 130, 132). Montserrat Roig, con su palabra, vuela y planea ese descenso. Atiende al «...somni de col.lectivitat» imaginado por el urbanista mediante tales patios, a su manera de dar forma a «...un civilitzat sentit del que ha de ser l'entesa col-lectiva» y de animar una nueva «àgora de la Mediterrània civilitzada» en clave ecléctica, entre Modernisme y Noucentisme; también a la destrucción ejecutada, con el tiempo, por los especuladores (Roig 1987: 158). Y de tal «paisatge urbà», de lo que, con todo, «...també és paisatge», conservando alguna que otra adelfa o palmera, entre el piar de los pájaros y el estruendo del tráfico callejero (Roig, 1987: 168, 158), la escritora rescata las «galeries» circundantes de tales espacios, abiertas en un tiempo, nos dice, en que el «balcó» en el extremo opuesto de la vivienda, en la fachada externa, se había transformado en «...reducte de les criades quan feien dissabte»(Roig 1987: 157-158). Esas galerías acristaladas, de interiores luminosos y calidos, hechas para compartir una privacidad moderna o, si se quiere, excluyente, hace tiempo que Carme Riera - glosando este texto de Montserrat Roig o tal vez otro con puntos de atención parejos, el titulado «De finestres, balcons i galeries» (AA.VV. 1989: 159-187) —nos las destacara como espacio femenino de la casa familiar; reducto confortable donde se llevaran a cabo las ocupaciones domésticas más artesanales y se deslizaran las plácidas horas menos hacendosas, entre la conversación, la lectura y, porqué no, la escritura: gineceo urbano donde creciera la palabra femenina a favor de la expresión de la cultura de la mujer; reducto que, de Vernet a Roig- generacionalmente pasando por la promoción intermedia de escritoras que se puede mirar en una Maria Aurèlia Capmany, para quien Barcelona era una ciudad a la vez «Vulnerable y consciente de sí misma...» y quien cuestiona su femenina identificación, según Maragall, con una «menestrala» (Capmany 1990: 107, 115-118)—, podemos pensar que no dejaría de ser mirado como una habitación propia en proceso, si se nos permite volver, con una ligera licencia, al término woolfiano. Pero nos place atender a la relevancia que a ese espacio le es concedida por la voz de una mujer que ya ha leído, pisado y dominado su ciudad hasta ser su memoria; apreciamos que la palabra de la mujer que ya puede volar libremente so- 
bre el cuerpo recostado de Barcelona haya vuelto sobre un punto concreto donde fue creciendo la palabra femenina. Atiéndase, además, que Montserrat Roig no desestima aquel otro espacio femenino doméstica y culturalmente contrapuesto donde hablan otras mujeres - el balcón con las criadas- y que ya no es el reducto solitario del primer texto tratado en estas páginas. Y aún, en su vuelo sobre Barcelona, la malograda escritora no olvida los jardincillos de las viviendas unifamiliares de Sarriá y de Sants (Roig 1987: 158), mención que debiera llevarnos al mundo entre verbal y vegetal de Mercè Rodoreda... Así creció, dejémoslo aquí, la polifonía femenina que finalmente ocupara Barcelona, mirada y pronunciada desde los espacios urbanos que la mujer fuera ganando: balcón, galería, jardín, calle, cielo...

5. Maria. Mercè Marçal (1952-1998), ‘Avui, vint-i-u de desembre...', Bruixa de dol (1977-1979).

Avui, vint-i-u de desembre,

he sortit al balcó:

sota una pluja que ja amainava

he vist els testos, les olles, els pots

de conserva plantats d'atzavares,

de cintes, begònies, geranis i cactus,

de cabellera de la reina

i d'alegria de la casa.

I el gesamí, que si se'm mor, no se'm mor.

Feia molts dies que no sortia al balcó

Corrent darrere d'amors i d'altres coses...

(Marçal 1989: 95)

Poeta y prosista, compañera de promoción literaria de las escritoras introducidas en el punto anterior, es seguramente la autora lírica con más reconocimiento por parte de crítica y de lectores en el inmediato cierre de siglo. MariaMercè Marçal, poeta barcelonesa, verdad es que la espacialidad de su escritura no suele medirse mediante referentes toponímicos, ciertamente escasos entre sus versos. En esa línea, la propia Barcelona no es una excepción. No obstante y acallando en este momento el interés que suscita la obra de Maria-Mercè Marçal en planos más trascendentes, nos permitiremos situar en la fachada del que era su domicilio en el momento de escribir el anterior poema —en Sant Boi, antes de sus posteriores mudanzas al Eixample, República Argentina y Passeig de Sant Joan- el «balcó» de los versos transcritos, aquel al que se asoma en la fecha que enuncia el primer verso del poema arriba mencionado.

La poeta sale a tal balcón en un día puntual, llevada por su personal decisión y para reencontrarse con su edén crecido sobre tiestos, pucheros y latas de conserva; cacharros de jardinera doméstica donde ha plantado las más variadas pitas, tiras, begonias, geranios, cactus y algazules, allí donde crece el jazmín, haciendo equilibrios entre aires de copla popular, si se mиеre, si nо se тиеге..., 
jardín aéreo que impone la alegría en su casa... La dueña y poeta de la casa sale a su balcón, lo hace bajo una lluvia que ya amaina, sosegadora, refrescante. Regresa a su lugar tras una larga e intensa ausencia de la que da puntual noticia. Lo hace tras haber estado "corrent darrere d'amors i d'altres coses...» (Marçal 1989: 95). Pero puede volver a la paz de su pequeño jardín, a la paz consigo misma después de haber vivido su ciudad, en la calle, corriendo tras sus más variados anhelos; entonces, ese balcón, parte de su vida y de su ciudad, ya no es un cerco excluyente por íntimo que sea. Ya no es el balcón oxigenante pero reductor de la Antonieta de Montserrat Monserdà. Su balcón y las calles por donde ha corrido forman parte de la cartografía urbana de las idas y venidas de la Marina y la Isabel de Maria Teresa Vernet. Y la barandilla que rodea al yo poético de Maria-Mercè Marçal entre sus plantas, sobrevuela la misma realidad humana que Montserrat Roig, la ciudad de Barcelona ya plenamente poseída por la mujer.

\section{BIBLIOGRAFÍA}

AA.VV. (1989): Barcel/dones, Barcelona, Edicions de l'Eixample.

CATAlÀ, Víctor (1985): Un film (3000 metres), Barcelona, Ed. 62 / La Caixa.

CAPMAnY, Maria Aurèlia (1990): ¿Qué diablos es Cataluña? Ser catalán hoy, Madrid, Ediciones Temas de Hoy.

Maragall, Joan (1970): Obres completes. Obra catalana, pròleg de Josep Carner, Barcelona, Editorial Selecta.

MARÇAL, Maria-Mercè (1989): Llengua abolida (1973-1988), València, Eliseu Climent, Editor.

MONSERdÀ, Dolors (1992): La fabricanta. Novel-la de costums barcelonines (18601875), introducció de Teresa Pàmies, Barcelona, Edicions de l'Eixample.

QuART, Pere (1979): Antologia, pròleg de Joan Triadú, Barcelona, Edicions Proa

RoIG, Montserrat, MisERACHS, Xavier (1987): Barcelona a vol d'ocell, Barcelona, Edicions 62.

VERDAGUER, Jacint (1974): Obres completes, pròleg de Marià Manent, epíleg de Joan Bonet i Baltà, Barcelona, Ediorial Selecta.

VERnET, Maria Teresa (2006): Les algues roges, introducció de Maria Campillo, Barcelona, Horsori. 